\title{
Impaired fasting glycaemia in children with obesity; should WHO or ADA cut-offs be used?
}

Emilia Hagman, Anna Ek, and Claude Marcus

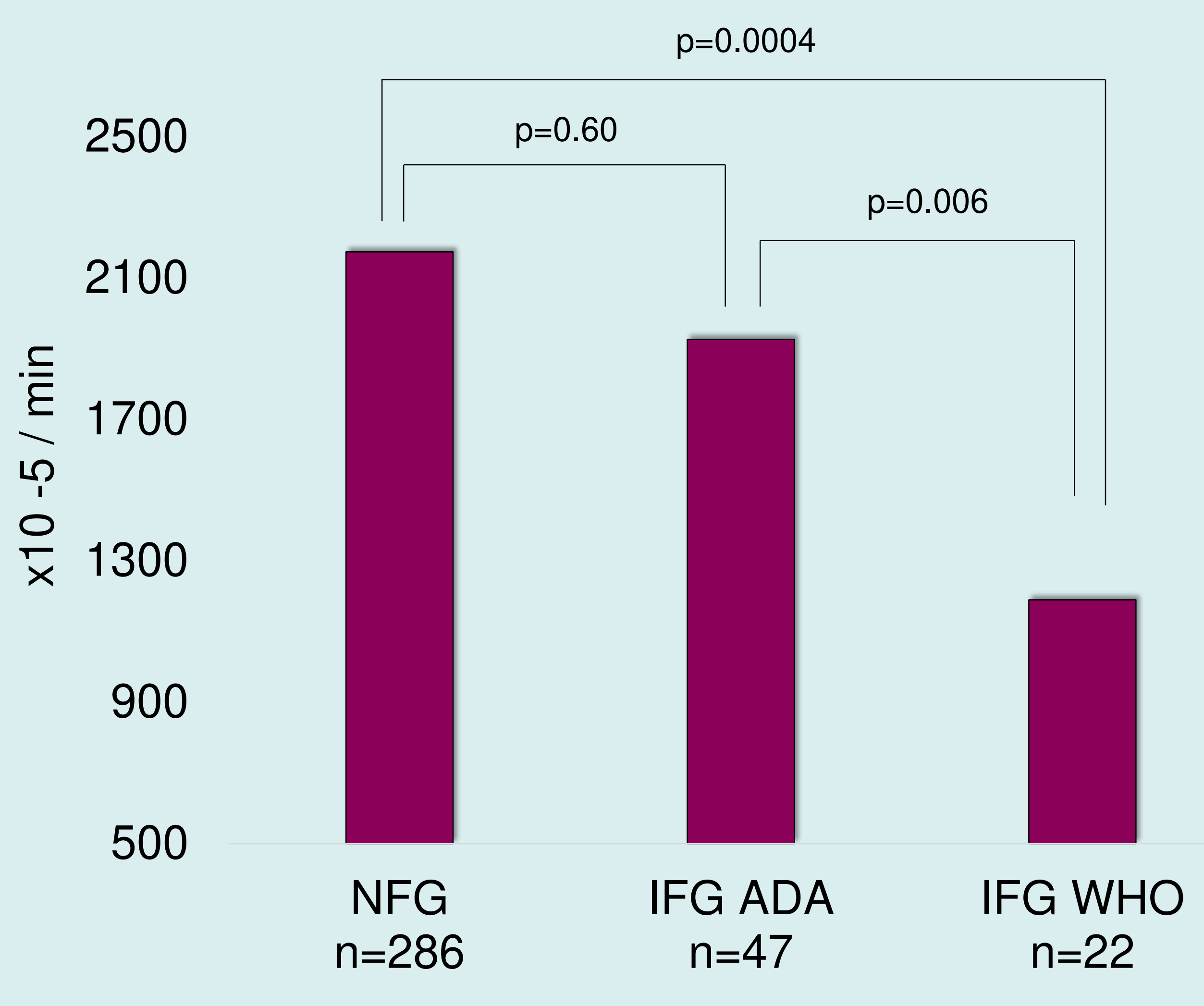

Subjects with IFG according to WHO, but not ADA, has a lower disposition index. Bars present median values. $\mathrm{NFG}=$ Normal fasting glycemia

\section{Conclusion}

Insulin resistance and disposition index is associated with IFG according to WHO, but not the exclusive range for IFG defined by ADA, in children and adolescents with obesity.

Using the ADA definition for IFG (part of current definitions of the metabolic syndrome) is possibly overestimating the prevalence of metabolic dysfunction in children and adolescents with obesity.

In combination with our previous research, IFG according to ADA not increases the risk for T2D more than childhood obesity itself, this clearly indicates that the prediabetic cut-off for IFG in childhood should be 6.1 $\mathrm{mmol} / \mathrm{L}(110 \mathrm{mg} / \mathrm{dL})$.

\section{Background}

Impaired fasting glucose (IFG), reflects impaired glucose-insulin homeostasis, and thereby associated with high risk of diabetes development. At present, two different cut-off values for IFG are used in parallel; the ADA suggest $5.6 \mathrm{mmol} / \mathrm{L}$ and the $\mathrm{WHO}$ promotes 6.1 $\mathrm{mmol} / \mathrm{L}$ as the cut-off for IFG.

We have recently shown that IFG according to the WHO definition, but not according to the exclusive interval for ADA (5.6-6.1 mmol/L), in pediatric obesity increases the risk for early adult T2DM.

\section{Aim}

Parameters of glycemic dysfunction include insulin resistance, disposition index and glucose effectiveness which all are risk factors for diabetes. To which extent the degree of these parameters are associated with the definitions of IFG in a pediatric population with obesity is yet to be determined.

\section{Methods}

Included in this study are children and adolescents ( $n=371,52 \%$ girls) with obesity according to International Obesity Task Force, between 5-18 years of age who have undergone an insulin modified intravenous glucose tolerance test at the National Childhood Obesity Centre, Sweden.

\section{Results}

The mean $\pm S D$ BMI SDS was $3.1 \pm 0.4$. The median \pm IQR age was $15.0 \pm 3.4$ years. The prevalence of IFG according to $\mathrm{WHO}$ was $7.0 \%$ and in the exclusive ADA range $12.9 \%$, with no sex differences, $p=0.3$ and $p=0.2$ respectively.

Insulin resistance was associated with IFG according to WHO compared with normal fasting glucose levels (adjusted OR=1.66 [1.06-2.58], $\mathrm{p}=0.03$ ), whereas the glucose range exclusive for the ADA definition was not associated with insulin resistance (adjusted $\mathrm{OR}=1.16$ [0.93-1.45], $p=0.20)$.

The disposition index, which takes both insulin sensitivity an acute insulin response into consideration, was $44 \%$ lower in subjects with IFG WHO compared with normal fasting glucose levels, $p<0.0001$, but no differences was observed in subject within the exclusive ADA range compared with normal fasting glucose levels, $p=0.60$.

The glucose effectiveness, a measure of how glucose modulates its own production, was $29 \%$ lower in subjects with IFG WHO compared with lower fasting glucose levels, $p=0.01$, but no differences was observed in subject within the exclusive ADA range, $\mathrm{p}=0.19$.

Exclusive IFG ADA $\quad 5.6-6.0 \mathrm{mmol} / \mathrm{L}$ or $100-110 \mathrm{mg} / \mathrm{dL}$ IFG WHO $\quad 6.1-6.9 \mathrm{mmol} / \mathrm{L}$ or $110-125 \mathrm{mg} / \mathrm{dL}$ 